\title{
La salud en el debate online: análisis de comentarios sobre carnes rojas y procesadas y cáncer en elpais.com
}

\section{Health in the online debate: analysis of comments about red and processed meats and cancer in elpais.com}

\author{
Cristina González-Pedraz; Ana-Victoria Pérez-Rodríguez
}

Cómo se cita este artículo:

González-Pedraz, Cristina; Pérez-Rodríguez, Ana-Victoria (2019). “La salud en el debate online: análisis de comentarios sobre carnes rojas y procesadas y cáncer en el elpais.com”. El profesional de la información, v. 28, n. 2, e280211.

https://doi.org//10.3145/epi.2019.mar.11

Artículo recibido el 10-09-2018

Aceptación definitiva: 31-01-2019

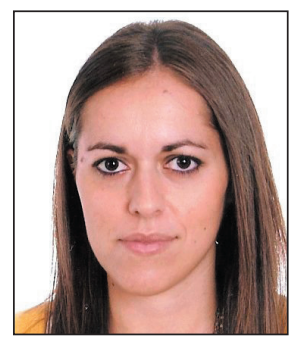

\section{Cristina González-Pedraz $\square$ http://orcid.org/0000-0001-8962-9224}

Fundación Centro de Estudios para la Ciencia, la Cultura Científica y la Innovación (3CIN) Ribera del Puente, 24, bajo. 37008 Salamanca, España cgonzalez@3cin.org

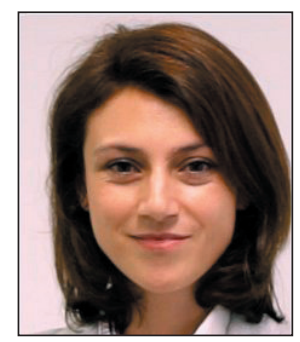

Ana-Victoria Pérez-Rodríguez http://orcid.org/0000-0003-3608-1765

Fundación Centro de Estudios para la Ciencia, la Cultura Científica y la Innovación (3CIN) Ribera del Puente, 24, bajo. 37008 Salamanca, España anavic@3cin.org

\section{Resumen}

En internet el lector puede participar en la información periodística a través de los comentarios, que se han convertido en una interesante fuente de datos para analizar la opinión de las audiencias sobre la actualidad científica y sanitaria. El presente trabajo realiza un análisis de contenido de un corpus de 2.394 comentarios a noticias sobre la relación entre carnes rojas y procesadas y cáncer en elpais.com. Los comentarios no parecen contribuir a una versión más completa del reporte periodístico, sino que el debate se desvirtúa en gran medida hacia otros asuntos. Los comentaristas se basan en su experiencia personal y en estereotipos para rechazar la evidencia científica.

\section{Palabras clave}

Periodismo digital; Ciberperiodismo; Periodismo científico; Periodismo de salud; Información de salud; Comentarios; Análisis de contenido; Nutrición; Carne; Cáncer; El país.

\begin{abstract}
On the Internet, the reader can participate in journalistic information through comments, which have become an interesting source of data to analyze the opinion of the audiences on current scientific and health. The present work carries out a content analysis of a sample of 2,394 news comments about the relationship between red and processed meats and cancer in elpais.com. The comments do not contribute to a more complete version of the journalistic report. The debate is distorted towards other issues. Commenters rely on their personal experience and stereotypes to reject scientific evidence.
\end{abstract}

\section{Keywords}

Digital journalism; Cyberjournalism; Science journalism; Health journalism; Health information; User comments; Content analysis; Nutrition; Meat; Cancer; El país. 


\section{Introducción}

Internet ha facilitado el acceso a información sobre ciencia y salud por parte del público (Hermida, 2010). El público recurre cada vez más a los cibermedios para seguir los avances científicos y participa en los medios digitales gracias a las actuales formas de comunicación dinámicas e interactivas, lo que para algunos autores lleva a reconfigurar y extender el concepto de audiencia en la Red (Holliman et al., 2009; Brossard, 2013).

De este modo el tradicional papel del receptor, limitado a leer, escuchar o visionar mensajes periodísticos de forma pasiva, ha dado un vuelco a partir de la web 2.0 (Reader, 2012), siendo reemplazado por una participación activa (Revuelta; Corchero-García, 2010; Laslo; Baram-Tsabari; Lewenstein, 2011; Blanchard, 2011; Cacciatore et al., 2012; Jang, 2013). El usuario puede realizar comentarios, críticas y sugerencias (Mauranen, 2013), valorar y compartir contenidos e incluso crear los suyos propios (Holliman et al., 2009; Blanchard, 2011; Veltri, 2013).

Utilizando como fuente de datos una de estas herramientas participativas, los comentarios a noticias, el presente trabajo pretende caracterizar el debate online en torno a una advertencia sanitaria que ha generado especial controversia en los últimos años. El 26 de octubre de 2015, el Centro Internacional de Investigaciones sobre el Cáncer (CIIC), entidad ligada a la Organización Mundial de la Salud (OMS), dio a conocer los resultados de una revisión de estudios científicos en torno a la posible carcinogenicidad del consumo de carne roja y carne procesada. El grupo de trabajo que realizó el informe, constituido por 22 expertos de 10 países, catalogó el consumo de carne roja como "probablemente carcinógeno" para los humanos, y la carne procesada como "carcinógena", una asociación que se observó, principalmente y en ambos casos, en el cáncer colorrectal ${ }^{1}$.

La cercanía a los ciudadanos de los resultados de este estudio, que cuestiona unos hábitos dietéticos arraigados entre la población española, ha originado que sea un asunto ampliamente comentado y discutido a nivel social, político y económico en España, constituyendo un objeto de investigación de interés en torno al cual hasta la fecha no se han localizado otros trabajos.

\subsection{Participación en la información a través de comentarios}

Diversos autores han estudiado, a nivel teórico y empírico, la participación de los usuarios mediante comentarios en el actual contexto de la información digital. Para Shanahan (2010), los comentarios online abren nuevas posibilidades en la comunicación de la ciencia y la salud: una interacción entre periodistas y usuarios donde el reconocimiento del status se "negocia" y en parte es determinado por las reacciones de los demás participantes.

Los comentarios también han abierto líneas de comunicación directas con los propios científicos. La sección de comentarios de los cibermedios presenta una oportunidad para que aquellos que no tienen acceso a los foros especializados puedan expresar su opinión. Las dinámicas de interacción entre estos agentes constituyen una línea de investigación de interés (Shanahan, 2010; Dunwoody, 2014).

Ante este panorama, Hermida (2010) considera que una tarea de los periodistas debe ser aportar valor añadido a la información científica a través de los nuevos sistemas participativas. Secko et al. (2011) profundizan en la idea de relato periodístico "inacabado", en referencia a las posibilidades de interacción con los lectores posteriores a la publicación de una pieza periodística en un cibermedio. Estos autores detectan una apertura de la narrativa del periodismo científico y sanitario online a través de la interacción que facilitan los comentarios, por ejemplo, cuestionando la autoridad del periodista o sus prácticas, contestando a las fuentes, reinterpretando la información o proporcionando sus propios consejos. Es decir, los comentarios favorecerían un reenfoque de los contenidos periodísticos relacionados con la ciencia y la salud.

Para algunos autores este reenfoque puede derivar en la distorsión de la información científica y constituiría uno de los principales peligros percibidos por los periodistas en relación con la sección de comentarios (Secko et al., 2011; Fernández-de-Lis, 2013). Otros autores tienen una visión más optimista y creen en la naturaleza auto-correctora de la Web y en que los lectores tienden a señalar los errores e imprecisiones de la información, promoviendo una comunicación periodística más fructífera (Pearce et al., 2015; Hermida, 2010).

Jaspal, Nerlich y Koteyko (2012) consideran además que las secciones de comentarios permiten la difusión de múltiples representaciones sociales y son un lugar para la contestación social y política, ya que pueden ser anónimos, posibilitan la interacción con los demás usuarios, tienen el potencial de influir en los demás participantes y pueden reflejar creencias colectivas y representaciones sociales predominantes. Por ello consideran que los comentarios son útiles para explorar cómo los lectores, a través de sus lentes culturales, perciben la relación entre ciencia, individuo y sociedad (Len-Ríos; Bhandari; Medvedeva, 2014).

Algunas investigaciones empíricas han ahondado en la narrativa de los comentarios a noticias relacionadas con ciencia y salud, utilizándolos como fuente de datos para conocer cómo determinados asuntos controvertidos son concebidos e interpretados por los lectores (Glenn; Champion; Spence, 2012; Holton; Lee; Coleman, 2014; Lei et al., 2015; Feinberg et al., 2015; Giles et al., 2015; Gardner; Smith; Mansfield, 2017; Fleming et al., 2017). 
También son relativamente frecuentes los estudios de comentarios a noticias sobre otras materias afines, como el medio ambiente. Abundan los estudios de caso en torno al debate generado por las informaciones sobre cambio climático (Jaspal; Nerlich; Koteyko, 2012; Koteyko; Jaspal; Nerlich, 2013; Collins; Nerlich, 2015). En estos trabajos se evidencia, entre otros aspectos, una marcada polarización del discurso, líneas de argumentación y opinión homogéneas o el afianzamiento de estereotipos científicos y políticos.

Otros estudios sugieren que los usuarios tienden a comentar más sobre su experiencia personal que sobre la evidencia científica (Shanahan, 2010; Secko et al., 2011; Len-Ríos; Bhandari; Medvedeva, 2014) y que la experiencia de las fuentes de información tiene una influencia significativa en la credibilidad del artículo reflejada en los comentarios de los lectores (Pjesivac; Geidner; Cameron, 2018).

Finalmente, uno de los aspectos más señalados en la bibliografía es la importante presencia de comentarios incívicos e insultantes en las noticias online sobre ciencia y salud (Secko et al., 2011; Brossard, 2013; Peters et al., 2014).

\subsection{Objetivos, preguntas de investigación e hipótesis}

Los estudios mencionados avalan que los comentarios realizados a noticias online constituyen una interesante fuente de datos para conocer la opinión de los usuarios sobre determinados temas de interés relacionados con la ciencia y la salud. Estas dos materias suelen estar estrechamente relacionadas, ya que las novedades reportadas en las noticias de salud son, en muchas ocasiones, resultado de estudios y avances científicos.

En este contexto, el objetivo principal del presente trabajo es caracterizar el debate online en torno a la relación entre carnes rojas y procesadas y cáncer. Se plantea el análisis de los comentarios generados por las noticias online sobre esta alerta sanitaria con el objetivo de extraer y caracterizar opiniones y encuadres discursivos prevalentes, el grado de contestación científica y posibles apelaciones al periodista autor de la información.

En concreto, se pretende responder a las siguientes preguntas de investigación:

P1: ¿Las aportaciones realizadas por los comentaristas son respetuosas y están fundamentadas en nuevas fuentes o datos de interés?

P2: ¿Qué recursos discursivos emplean los comentaristas?

P3: ¿Se está produciendo una contestación científica a través de los comentarios online?

P4: ¿Los comentarios apelan de alguna forma al autor de la información?

Partiendo de los resultados reportados en estudios previos, se formulan las siguientes hipótesis:

H1: los comentarios sobre carnes rojas y procesadas y cáncer son generalmente respetuosos.

H2: la argumentación no se asienta sobre nuevas fuentes o datos de interés adicionales.

H3: los comentaristas aluden a la experiencia personal, al humor y a estereotipos cuando debaten respecto al objeto de estudio.

H3: los comentarios se utilizan como medio de contestación científica.

H4: se apela frecuentemente a la figura del periodista autor de la información, principalmente en un tono negativo.

\section{Metodología}

Se plantea un análisis de contenido de comentarios realizados a noticias sobre carnes rojas y procesadas y cáncer publicadas en Elpaís.com ${ }^{2}$ desde que se emitiera la alerta sanitaria, el 26 de octubre de 2015, hasta el 1 de febrero de 2018. La búsqueda de las noticias se realizó de forma manual a través de la hemeroteca del periódico, utilizando las siguientes cadenas de palabras clave:

- "carne roja"+cáncer;

- "carne procesada"+cáncer;

- carne+roja+procesada+cáncer;

- "carnes rojas"+cáncer;

- "carnes procesadas"+cáncer;

- carnes+rojas+procesadas+cáncer.

La búsqueda se llevó a cabo el 2 de febrero de 2018, localizándose 27 noticias. Seis de ellas no presentaron comentarios, mientras que las 21 restantes registraron 2.394 comentarios, que conformaron la muestra final.

Posteriormente se elaboró una ficha de análisis y un libro de códigos compuesto por una batería de variables de tipo cualitativo diseñada a partir de las aportaciones de otros autores y complementada por las autoras. Para categorizar algunas de las variables exploradas, las relativas a los encuadres discursivos [tabla 1, dimensión 3: Encuadres generales], se ha aplicado la teoría del frame (Scheufele, 1999; Bubela et al., 2009; Koziner, 2013; Ardèvol-Abreu, 2015). 
Posteriormente se probó la utilidad del libro de códigos y la fiabilidad de las dos codificadoras -las autoras del trabajoen una muestra aleatoria de 50 unidades de análisis (comentarios). Este ensayo se llevó a cabo entre el 5 y el 7 de febrero de 2018. Se obtuvo un coeficiente de fiabilidad satisfactorio (94,4\%) según las estimaciones de Lombard, Snyder-Duch y Bracken (2002) y se actualizó el libro de códigos, formulando nuevas respuestas para algunas de las variables. La ficha de análisis se completó con una pregunta abierta para recoger observaciones y/o reflexiones de los codificadores acerca de los comentarios.

\section{Resultados}

Se presentan los resultados del análisis en tres apartados que se corresponden con los objetivos del estudio y que tratan de responder a las preguntas de investigación planteadas.

Tabla 1. Ficha de análisis aplicada. Variables y tipos de respuesta.

\begin{tabular}{|c|c|c|}
\hline \multicolumn{2}{|r|}{ Variable } & Tipo de respuesta \\
\hline \multicolumn{3}{|c|}{ 1. Datos identificativos de la noticia } \\
\hline 1.1. & Titular de la noticia & \\
\hline 1.2. & URL & \\
\hline 1.3. & Fecha & DD/MM/AAAA \\
\hline 1.5. & Número total de comentarios & \\
\hline \multicolumn{3}{|c|}{ 2. Datos identificativos del comentario } \\
\hline 2.1. & Número de unidad de análisis (número asignado al comentario) & \\
\hline 2.2. & Número de comentario (asignado por elpais.com) & \\
\hline 2.3. & Comentario & \\
\hline 2.4. & Mes y año & MM/AAAA \\
\hline \multicolumn{3}{|c|}{ 3. Encuadres generales } \\
\hline 3.1. & $\begin{array}{l}\text { Aportación constructiva (el texto permite extraer opiniones respe- } \\
\text { tuosas) }\end{array}$ & $\begin{array}{l}\text { Sí } \\
\text { No } \\
\text { No aplica }\end{array}$ \\
\hline 3.2. & $\begin{array}{l}\text { Fuentes (se observa una documentación del comentarista que } \\
\text { aporta nuevas fuentes o datos de interés) }\end{array}$ & $\begin{array}{l}\text { Sí } \\
\text { No }\end{array}$ \\
\hline 3.3. & $\begin{array}{l}\text { Errores (el comentarista señala, de forma equivocada o no, errores } \\
\text { y/o imprecisiones del contenido periodístico que comenta, según } \\
\text { la idea de la naturaleza autocorrectora de la Web, Hermida, 2010) }\end{array}$ & $\begin{array}{l}\text { Sí } \\
\text { No }\end{array}$ \\
\hline 3.4. & Humor (el comentario es sarcástico, irónico o emplea el humor) & $\begin{array}{l}\text { Sí } \\
\text { No }\end{array}$ \\
\hline 3.5. & $\begin{array}{l}\text { Experiencia personal (en las aportaciones respetuosas el comen- } \\
\text { tarista habla más de su experiencia personal que de la evidencia } \\
\text { científica, de acuerdo con Shanahan, } 2010 \text { y Len-Ríos; Bhandari; } \\
\text { Medvedeva, 2014) }\end{array}$ & $\begin{array}{l}\text { Sí } \\
\text { No } \\
\text { No aplica }\end{array}$ \\
\hline 3.6. & $\begin{array}{l}\text { Estereotipos (el comentario refleja estereotipos científicos y socia- } \\
\text { les, en línea con el estudio de Koteyko; Jaspal; Nerlich, 2013) }\end{array}$ & $\begin{array}{l}\text { Sí } \\
\text { No }\end{array}$ \\
\hline \multicolumn{3}{|c|}{ 4. Contestación científica } \\
\hline 4.1. & Acuerdo/desacuerdo científico & $\begin{array}{l}\text { Acuerdo = manifiesta un apoyo expreso } \\
\text { Desacuerdo = manifiesta un desacuerdo expreso } \\
\text { Dos versiones = sopesa argumentos a favor y en contra } \\
\text { Otros } \\
\text { No aplica }\end{array}$ \\
\hline 4.2. & Tipo de crítica & $\begin{array}{l}\text { Argumentada = realiza una crítica argumentada } \\
\text { Negación = realiza una negación pura y simple del fenómeno } \\
\text { Deslegitimación = denigra/deslegitima a la fuente experta } \\
\text { Otros } \\
\text { No aplica }\end{array}$ \\
\hline \multicolumn{3}{|c|}{ 5. Apelación al periodista } \\
\hline 5.1. & Tipo de apelación predominante & $\begin{array}{l}\text { Autoridad = cuestiona la autoridad del periodista y/o sus prácticas } \\
\text { Fuentes = cuestiona las fuentes utilizadas } \\
\text { Errores = señala errores o imprecisiones } \\
\text { Insulto = Realiza una crítica no constructiva y/o insultante } \\
\text { Felicitación = felicita al periodista por su trabajo } \\
\text { Otros } \\
\text { No aplica }\end{array}$ \\
\hline
\end{tabular}




\title{
3.1. Encuadres generales
}

Las aportaciones realizadas por los comentaristas son respetuosas en un $61,07 \%(n=1462)$ de los casos. En tan sólo un $12,45 \%(n=298)$ de los comentarios se observa una documentación por parte del usuario, aportando nuevas fuentes o datos de interés. En un 6,77\% ( $n=162)$ de los comentarios el usuario señala, de forma equivocada o no, errores o imprecisiones del contenido periodístico que comenta.

\begin{abstract}
"La Sociedad Española de Nutrición ha contestado a la OMS que la carne roja tiene beneficios nutricionales y que aporta vitaminas, proteínas, hierro y zinc. Y que el jamón forma parte de la mundialmente reconocida dieta mediterránea, que fue declarada Patrimonio Cultural de la Humanidad por parte de la Unesco. Los españoles podemos estar muy tranquilos porque nuestro consumo de carne roja y procesada es inferior a lo señalado como peligroso por la OMS. Cada ciudadano consume de media un kilo de carne a la semana, lo que supone 50 kilos al año, de los que 15 kilos son de pollo, 12 kilos de cerdo y 6 kilos de vacuno. Un español consume 350 gramos de carne roja a la semana (cifra que está por debajo de los 500 gramos máximos que recomienda la OMS) y 30 gramos diarios de carne procesada, que también es inferior a los 50 gramos que esta organización califica como peligroso".
\end{abstract}

Comentario n. 1.672. Ejemplo de aportación constructiva cuya argumentación está documentada. Recuperado el 02/02/2018 de:

http://ow.ly/c2QK30kTMMJ

El humor y la ironía se utilizan en el $28,28 \%(n=677)$ de los comentarios.

\begin{abstract}
"En un alarde de altruismo y después del demoledor informe de la OMS he creado un servicio gratuito de recogida de jamones y chuletones de ternera, residuos en definitiva... para proceder a su procesamiento y destrucción con garantía ya que en casa cuento con un equipo humano altamente especializado. Gracias. PD: da igual si el jamón ya está empezado".
\end{abstract}

Comentario n. 1.344. Ejemplo del uso del humor en el debate sobre ciencia y salud online. Recuperado el 02/02/2018 de http://ow.ly/5ezV30kTR5h

Por otra parte, más de la mitad de los comentaristas $(52,44 \% ; n=1255)$ comparte sus experiencias y vivencias personales y se basa en ellas para apoyar o rechazar la evidencia científica reportada.

\footnotetext{
"Luego uno lee la noticia de más abajo y todo queda, cuando menos, confuso porque no queda para nada claro si es bueno o no comer carne. Yo sólo sé que desde pequeño como hamburguesas, salchichas y embutidos, me hice una colonoscopia y una endoscopía el año pasado y nada de nada. Claro tampoco me inflé pero lo comía relativamente seguido. No sé yo si estos estudios hay que darles mucha credibilidad. Por cierto, mi edad actual son 47 años".

"Bueno yo lo sé por experiencia propia, la mayor parte de vecinos y gente conocida que vivían en mi pueblo se atiborraban de embutidos y cosas procesadas (además de fumar y beber alcohol) y ninguno llego a los 60 años, algunos ni a los 50".
}

Comentarios n. 322 y 1.123 . Ejemplos de apelación a la experiencia personal para apoyar o no los argumentos científicos. Recuperados el 02/02/2018 de:

http://ow.ly/91wH30kTRvp

Por último, la referencia a estereotipos en los comentarios analizados se registra en casi la mitad de la muestra (46,99\%; $\mathrm{n}=1125)$.

"Cuando era un adolescente mis compañeros de secundaria se reían de mi vegetarianismo. Hoy, con 55 años de edad, me los encuentro por ahí de vez en cuando con todo tipo de problemas de salud, mientras que se asombran de mi nivel de energía, salud y figura. Tú quédate con tu humor y tus intestinos repletos de carne a medio digerir. Yo seguiré con los cientos de deliciosas recetas vegetarianas disponibles en el mundo".

“No sé... por aquí hay más de un/una arrogante moralista que desde lo alto de su ego vegano superior parece que la prohibiría... Creo que aquí no se trata de salvar el planeta o a los animales sino más bien de cultivar y regodearse en una superioridad moral imaginaria".

Comentarios n. 610 y 614. Ejemplos del uso de estereotipos. Recuperados el 02/02/2018 de:

http://ow.ly/91wH30kTRvp

\subsection{Contestación científica}

En relación con esta dimensión, las dos variables medidas se han marcado con la respuesta 'no aplica' en un 73,31\% ( $\mathrm{n}$ = 1755) de los comentarios analizados, en los que el debate se desvía claramente del contenido de la noticia. Según los datos recogidos en la pregunta abierta, en todos los comentarios marcados como 'no aplica' el debate sobre la relación entre carnes rojas y procesadas y cáncer deriva hacia un enfrentamiento entre partidarios y detractores del veganismo. Se observa que organizaciones pro veganismo dan respuesta a usuarios particulares que atacan su filosofía, extendiendo, partidarios y detractores, un debate en gran medida ajeno a la noticia que comentan.

Los comentarios se utilizan como medio de contestación científica, en gran medida sustentada en la negación de la evidencia científica, sin aportar otros argumentos 
Entre los usuarios que sí debaten sobre el objeto de la noticia (el 26,69\% del total de comentarios; $n=639$ ), se observa una marcada polarización en el discurso. Esto se observa porcentualmente en un equilibrio entre aquellos que se muestran de acuerdo con la evidencia científica y respaldan los resultados del estudio y/o a los investigadores autores del mismo $(43,51 \% ; n=279)$, y los que están en desacuerdo (51,33\%; $n=328)$. Un $3,44 \%(n=22)$ de los comentaristas aporta argumentos a los dos lados de la balanza.

Del porcentaje de usuarios en desacuerdo, se tiende a negar la evidencia científica sin aportar ningún otro tipo de argumento en un 46,36\% (n = 152) de los casos. Otro modo de contestación científica, la deslegitimización tanto de los científicos como de la OMS como responsable del estudio y emisora de la alerta sanitaria, se observa en un $27,41 \%(n=70)$ de los comentarios en desacuerdo. Un $19,83 \%$ ( $n=65$ ) de los comentarios en desacuerdo aporta argumentos, de mayor o menor peso, para contrarrestar la evidencia científica.

\footnotetext{
"Pues mire, voy a seguir comiendo carne de lo que me parezca pese a lo que usted opine y de lo que opine la OMS".
}

Realiza una aportación constructiva

Aporta nuevas fuentes

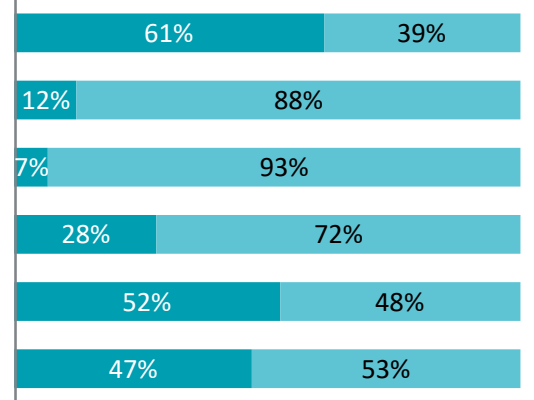

0\% 10\% 20\% 30\% 40\% 50\% 60\% 70\% 80\% 90\%100\%

$$
\text { - Sí } \square \text { No }
$$

Figura 1. Encuadres generales (Sí/No \%).

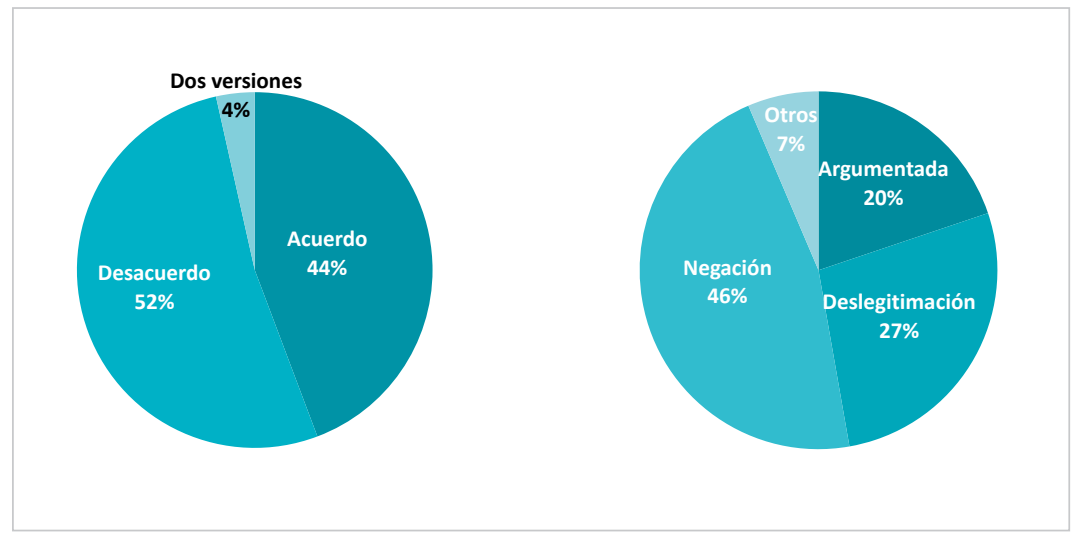

Figura 2. Acuerdo/desacuerdo con la evidencia científica manifestado (A) y tipo de crítica de los comentaristas que están en desacuerdo (B)

\begin{abstract}
"Según la OMS todos íbamos a morir de sida, después de hepatitis B, se sacrificaron cientos de miles de vacas innecesariamente, después de gripe asiática, aviar y por último de gripe A. Ahora la carne roja es la culpable de todos nuestros males. Si usted se cree lo que dice la OMS, vaya usted a vivir al pueblo de montaña más alejado de cualquier urbe, limítese a comer coles de Bruselas, pepino crudo, zanahorias y ocasionalmente calabacín. Cero tabaco, cero alcohol, y morirá de cualquier cosa menos de cáncer de colon. Morirá de hepatitis o de gripe $\mathrm{A}^{\prime \prime}$.

"Sabiendo que la probabilidad en España de padecer cáncer colorrectal es del 5\% aprox. (algo más en hombres que en mujeres) el consumo de embutidos provocaría que esta probabilidad pasara al 5,9\% ( $18 \%$ de $5 \%$ es $0,9 \%$ ). Lo que me parece una ridiculez en cuanto al número de factores que influyen en esta enfermedad. Por otra parte si miráis el artículo de The Lancet veréis cómo el $50 \%$ de los estudios no muestran esta correlación entre cáncer y carnes procesadas y además los estudios son epidemiológicos, es decir, de correlación, por tanto no dan ningún tipo de evidencia sino que predicen una posible implicación que debería probarse empíricamente, y en esto los estudios no son tan claros, nuevamente con estudios contradictorios. En definitiva, creo que la OMS se ha precipitado y que es una burrada incluir las carnes procesadas en el mismo grupo que otros cancerígenos plenamente probados".
\end{abstract}

Comentarios n. 1.061, 343 y 510. Ejemplos de tipos de contestación científica: negación, deslegitimación y crítica argumentada. Recuperados el 02/02/2018 de:

http://ow.ly/FQSa3014xj6

\title{
3.3. Apelación al periodista
}

Los comentarios en los que se apela de algún modo al periodista autor de la información, son escasamente frecuentes $(4,22 \% ; n=101)$. De esta proporción de comentarios en los que se apela al periodista, en un $40,51 \%$ ( $n$ =41) de los casos se cuestiona su autoridad o sus prácticas profesionales. El 31,31\% ( $n=32$ ) señala, de forma acertada o no, errores o imprecisiones; y un $5,05 \%(n=5)$ realiza una crítica no constructiva o insultante.

En un $8,08 \%(n=8)$ de los casos el comentario es positivo hacia la labor del periodista. Según los datos recabados en la pregunta abierta, cuando se apela al periodista predomina un tono marcadamente negativo. Se ataca, por lo general, a su profesionalidad (falta de cualificación, falta de comprensión de la información reportada, etc.) y a sus prácticas (mala redacción del artículo, fuentes no contrastadas, etc.). 


\begin{abstract}
"Las noticias sobre ciencia de este diario siempre van pobremente redactadas porque son pésimas traducciones del inglés. Los reporteros deberían saber, por ejemplo, que organismos como la OMS publican los mismos informes en castellano bien escrito, no la lengua chapucera que nos asesta este diario".

"Totalmente de acuerdo con el artículo (tendríais que ver una respuesta que escribí ayer ante el pánico general de las redes sociales, ni que me la hubieseis calcado)... pero por favor... qué es eso de "linkado"... tenemos suficientes verbos en castellano... "enlazado", por Dios, que esto es un periódico nacional no una conversación de "hatsapp".
\end{abstract}

Comentarios n. 473 y 1.776. Ejemplos de apelación al periodista. Recuperados el 02/02/2018 de: http://ow.ly/FQSa30l4xj6

\title{
4. Conclusiones y discusión
}

Nuestro caso se centra por primera vez en el debate online sobre la relación entre carnes rojas y procesadas y cáncer, un asunto de actualidad que ha generado una importante alarma debido, fundamentalmente, a su cercanía a los ciudadanos. El trabajo confirma que las posibilidades que internet brinda para expresar opiniones en foros como las cibernoticias confieren a los comentarios un interés sustancial como fuente de datos para conocer cómo los lectores perciben y responden a los asuntos de actualidad científica y sanitaria.

El debate online sobre carnes rojas y procesadas y cáncer es respetuoso en más de la mitad de los comentarios analizados, a diferencia de los trabajos que advierten de la importante presencia de comentarios incívicos en estos foros (Secko et al., 2011; Brossard, 2013; Peters et al., 2014). Pese a ser un debate en general 'educado', los argumentos que se esgrimen no están

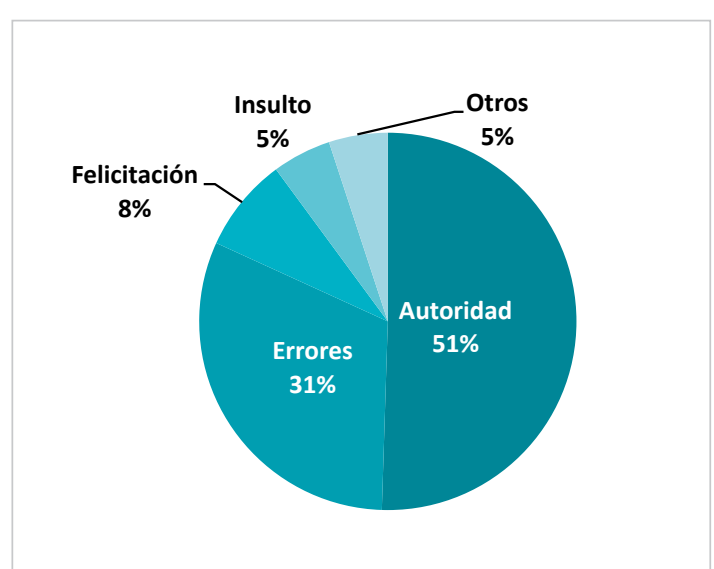

Figura 3. Tipo de apelación al periodista predominante. fundamentados, en prácticamente ningún caso, en fuentes complementarias a las aportadas por el periodista o en otros datos de interés. Así, los resultados permiten confirmar la H1: aunque en la teoría los comentarios permiten abrir al público la narrativa periodística, que se torna en relato "inacabado" en el contexto online (Secko et al., 2011), ésta no parece verse enriquecida por las aportaciones de los lectores, quienes no contribuyen efectivamente a generar una versión más completa del reporte periodístico.

Es significativo cómo los comentaristas hacen caso omiso a las fuentes expertas y se apoyan en su experiencia personal y en estereotipos, sin ningún fundamento, para cuestionar los argumentos científicos reportados. Esta situación se había observado en otros contextos (Koteyko; Jaspal; Nerlich, 2013; Len-Ríos; Bhandari; Medvedeva, 2014) y permite contrastar la $\mathrm{H} 2$.

\section{Se observa una marcada polarización en el discurso, así como argumentos y opi- niones homogéneas pro y en contra del comunicado de la OMS}

Uno de los resultados más destacados del análisis es que el 73,31\% de los comentarios se centra en un debate paralelo en torno al veganismo y su filosofía, que termina prácticamente fagocitando la discusión sobre el objeto de estudio. Así, los comentaristas parecen utilizar el contenido de una noticia como excusa para discutir sobre otros temas de su interés, en línea con lo sugerido por Robinson (2010) y Laslo, Baram-Tsabari y Lewenstein (2011).

Entre aquellos que sí debaten sobre la relación entre carnes rojas y procesadas y cáncer se observa una marcada polarización en el discurso, así como argumentos y opiniones homogéneas a ambos lados de la balanza, un aspecto que ya había sido advertido en otro estudio de caso (Collins; Nerlich, 2015). Tal y como preveía la H3, los comentarios se usan como medio de contestación científica, que en gran medida se sustenta en la simple negación de la evidencia científica, al igual que se ha registrado en trabajos previos (Jaspal; Nerlich; Koteyko, 2012).

Por último, los comentaristas apelan al periodista autor de la noticia en un escaso número de comentarios, por lo que se refuta la $\mathrm{H} 4$. En los casos en los que se alude a esta figura, el tono es muy negativo y se cuestionan tanto las capacidades del periodista como sus prácticas.

Este estudio tiene implicaciones en cómo el público comprende y se apropia de los principales problemas científicos y sanitarios del momento. No obstante, será necesario profundizar sobre los efectos que tienen los comentarios no fundamentados y las opiniones mal informadas en los lectores.

El caso analizado es sólo una instantánea sobre un asunto determinado en un período de tiempo concreto, dentro de un panorama online altamente cambiante. El estudio presenta algunas limitaciones, como el hecho de que se hayan analizado comentarios procedentes de un
La narrativa periodística sobre la relación entre carnes rojas y procesadas y cáncer no se enriquece con los comentarios de los lectores 
sólo medio de comunicación, o el sesgo que podría conllevar la fiabilidad de la codificación. Por ello, serán necesarios más estudios en torno a éste y otros temas, y en distintos medios de comunicación, para confirmar las tesis apuntadas en este trabajo y conocer cómo evoluciona el debate sobre ciencia y salud en internet.

\section{Notas}

1. Más información sobre el estudio del CIIC disponible en: http://ow.ly/KaP330kNvyo

2. Según el medidor de referencia ComScore, elpais.com es el periódico digital más leído en España según los últimos datos disponibles (febrero de 2018):

http://ow.ly/lgfX30kOkDZ

\section{Referencias}

Ardèvol-Abreu, Alberto (2015). “Framing o teoría del encuadre en comunicación. Orígenes, desarrollo y panorama actual en España". Revista latina de comunicación social, v. 70, pp. 423-450.

https://doi.org/10.4185/RLCS-2015-1053

Blanchard, Antoine (2011). "Science blogs in research and popularization of science: Why, how and for whom?". In: Cockell, Moira; Billotte, Jerôme; Darbellay, Fréderic; Waldvogel, Francis. Common knowledge: The challenge of transdisciplinarity. Lausanne: EPFL Press, pp. 219-231. ISBN: 9781439863312

https://www.archives-ouvertes.fr/hal-01249315/document

Brossard, Dominique (2013). "New media landscapes and the science information consumer". Proceedings of the National Academy of Sciences of the United States of America, v. 10, n. 3, pp. 14096-14101.

https://doi.org/10.1073/pnas.1212744110

Bubela, Tania; Nisbet, Matthew C.; Borchelt, Rick; Brunger, Fern; Critchley, Cristine; Einsiedel, Edna; Geller, Gail; Gupta, Anil; Hampel, Jürgen; Hyde-Lay, Robyn; Jandciu, Eric W.; Jones, S. Ashley; Kolopack, Pam; Lane, Summer; Lougheed, Tim; Nerlich, Brigitte; Ogbogu, Ubaka; O'Riordan, Kathleen; Ouellette, Colin; Spear, Mike; Strauss, Stephen; Thavaratnam, Thushaanthini; Willemse, Lisa; Caulfield, Timothy (2009). "Science communication reconsidered". Nature biotechnology, v. 27, n. 6, pp. 514-518.

http://doi.org/10.1038/nbt0609-514

Cacciatore, Michael A.; Anderson, Ashley A.; Choi, Doo-Hun; Brossard, Dominique; Scheufele, Dietram A.; Liang, Xuan; Ladwig, Peter J.; Xenos, Michael; Dudo, Anthony (2012). "Coverage of emerging technologies: A comparison between print and online media". New media \& society, v. 14, n. 6, pp. 1039-1059.

http://ow.ly/RxwT30iKB2y

https://doi.org/10.1177/1461444812439061

Collins, Luke; Nerlich, Brigitte (2015). “Examining user comments for deliberative democracy: A corpus-driven analysis of the climate change debate online". Environmental communication, v. 9, n. 2, pp. 189-207.

https://doi.org/10.1080/17524032.2014.981560

Dunwoody, Sharon (2014). "Science journalism: Prospects in the digital age". In: Bucchi, Massimiano; Trench, Brian. Routledge handbook of public communication of science and technology, pp. 27-39. Routledge. ISBN: 9780415834612 http://ow.ly/kb3130ikFuf

Feinberg, Yael; Pereira, Jennifer A.; Quach, Susan; Kwong, Jeffrey C.; Crowcroft, Natasha S.; Wilson, Sarah E.; Guay, Marise; Lei, Yang; Deeks, Shelley L. (2015). “Understanding public perceptions of the HPV vaccination based on online comments to Canadian news articles". PloS one, v. 10, n. 6.

https://doi.org/10.1371/journal.pone.0129587

Fernández-De-Lis, Patricia (2013). "Ciencia y periodismo en la Red”. Quaderns de la Fundació Dr. Antoni Esteve, v. 28, pp. 15-19.

http://ow.ly/WoDt30iLC8F

Flemming, Danny; Feinkohl, Insa; Cress, Ulrike; Kimmerle, Joachim (2017). "User comments about research findings: How conflictual information in online science journalistic articles influences lay people's understanding of scientific tentativeness". Communications, v. 42, n. 4, pp. 465-480.

https://doi.org/10.1515/commun-2017-0037

Gardner, Benjamin; Smith, Lee; Mansfield, Louise (2017). “How did the public respond to the 2015 expert consensus public health guidance statement on workplace sedentary behaviour? A qualitative analysis". BMC public health, v. 17, n. 1, pp. 47. https://doi.org/10.1186/s12889-016-3974-0

Giles, Emma L.; Holmes, Matthew; McColl, Elaine; Sniehotta, Falko F.; Adams, Jean M. (2015). “Acceptability of financial incentives for breastfeeding: Thematic analysis of readers' comments to UK online news reports". BMC pregnancy 
and childbirth, v. 15, n. 116.

https://doi.org/10.1186/s12884-015-0549-5

Glenn, Nicole M.; Champion, Claudine C.; Spence, John C. (2012). “Qualitative content analysis of online news media coverage of weight loss surgery and related reader comments". Clinical obesity, v. 2, n. 5-6, pp. 125-131.

https://doi.org/10.1111/cob.12000

Hermida, Alfred (2010). "Revitalizing science journalism for a digital age". In: Kennedy, Donald; Overholser, Geneva. Science and the media. Cambridge, MA: American Academy of Arts and Sciences, pp. 80-87. ISBN: 0877240876 https://www.amacad.org/publication/science-and-media

Holliman, Richard; Whitelegg, Elizabeth; Scanlon, Eileen; Smidt, Sam; Thomas, Jeff (2009). Investigating science communication in the information age: Implications for public engagement and popular media. Oxford University Press. ISBN: 9780199552665

Holton, Avery; Lee, Nayeon; Coleman, Renita (2014). "Commenting on health: A framing analysis of user comments in response to health articles online". Journal of health communication, v. 19, n. 7, pp. 825-837.

https://doi.org/10.1080/10810730.2013.837554

Jang, S. Mo (2013). "Seeking congruency or incongruency online? Examining selective exposure to four controversial science issues". Science communication, v. 36, n. 2, pp. 143-167.

https://doi.org/10.1177/1075547013502733

Jaspal, Rusi; Nerlich, Brigitte; Koteyko, Nelya (2012). “Contesting science by appealing to its norms: Readers discuss climate science in the Daily Mail”. Science communication, v. 35, n. 3, pp. 383-410.

http://ow.ly/aWHc30iMCqL

https://doi.org/10.1177/1075547012459274

Koteyko, Nelya; Jaspal, Rusi; Nerlich, Brigitte (2013). “Climate change and 'climategate' in online reader comments: A mixed methods study". The geographical journal, v. 179, n. 1, pp. 74-86.

http://ow.ly/LJif30iMDnb

https://doi.org/10.1111/j.1475-4959.2012.00479.x

Koziner, Nadia-Sabrina (2013). "Antecedentes y fundamentos de la teoría del framing en comunicación". Austral comunicación, v. 2, n. 1, pp. 01-25.

http://ow.ly/yxFV30iMDHK

Laslo, Esther; Baram-Tsabari, Ayelet; Lewenstein, Bruce V. (2011). "A growth medium for the message: Online science journalism affordances for exploring public discourse of science and ethics". Journalism, v. 12, n. 7, pp. 847-870.

https://doi.org/10.1177/1464884911412709

Lei, Yang; Pereira, Jennifer A.; Quach, Susan; Bettinger, Julie A.; Kwong, Jeffrey C.; Corace, Kimberly; Garber, Gary; Feinberg, Yael; Guay, Maryse (2015). “Examining perceptions about mandatory influenza vaccination of healthcare workers through online comments on news stories". PloS one, v. 10, n. 6.

https://doi.org/10.1371/journal.pone.0129993

Len-Ríos, María E.; Bhandari, Manu; Medvedeva, Yulia S. (2014). "Deliberation of the scientific evidence for breastfeeding: Online comments as social representations". Science communication, v. 36, n. 6, pp. 778-801.

https://doi.org/10.1177/1075547014556195

Lombard, Matthew; Snyder-Duch, Jennifer; Bracken, Cheryl C. (2002). "Content analysis in mass communication: Assessment and reporting of intercoder reliability". Human communication research, v. 28, n. 4, pp. 587-604.

https://engagedscholarship.csuohio.edu/cgi/viewcontent.cgi?article=1049\&context=clcom_facpub https://doi.org/10.1111/j.1468-2958.2002.tb00826.x

Mauranen, Anna (2013). "Hybridism, edutainment, and doubt: Science blogging finding its feet". Nordic journal of English studies, v. 13, n. 1, pp. 7-36.

http://ow.ly/n4kj30iOgZ6

Pearce, Warren; Brown, Brian; Nerlich, Brigitte; Koteyko, Nelya (2015). “Communicating climate change: Conduits, content, and consensus". Wiley interdisciplinary reviews: Climate change, v. 6, n. 6, pp. 613-626.

http://ow.ly/AOjm30iOpuF

https://doi.org/10.1002/wcc.366

Peters, Hans-Peter; Dunwoody, Sharon; Allgaier, Joachim; Lo, Yin-Yueh; Brossard, Dominique (2014). "Public communication of science 2.0: Is the communication of science via the 'new media' online a genuine transformation or old wine in new bottles?". EMBO reports, v. 15, n. 7, pp. 749-753.

http://ow.ly/AhMm30iOpXq 
https://doi.org/10.15252/embr.201438979

Pjesivac, Ivanka; Geidner, Nicholas; Cameron, Jaclyn (2018). "Social credibility online: The role of online comments in assessing news article credibility". Newspaper research journal, v. 39, n. 1, pp. 18-31.

https://doi.org/10.1177/0739532918761065

Reader, Bill (2012). "Free press vs. free speech? The rhetoric of 'civility' in regard to anonymous online comments". Journalism \& mass communication quarterly, v. 89, n. 3, pp. 495-513.

https://doi.org/10.1177/1077699012447923

Revuelta, Gema; Corchero-García, Cristina (2010). "Búsqueda activa y recepción pasiva de información sobre ciencia y tecnología". En: Percepción social de la ciencia y la tecnología 2010. Fundación Española para la Ciencia y la Tecnología (Fecyt), pp. 183-202.

http://ow.ly/UKOq30iOs58

Robinson, Sue (2010). "Traditionalists vs. convergers: Textual privilege, boundary work and the journalist-audience relationship in the commenting policies of online news sites". Convergence, v. 16, n. 1, pp. 125-143.

https://doi.org/10.1177/1354856509347719

Scheufele, Dietram A. (1999). "Framing as a theory of media effects". Journal of communication, v. 49, n. 1, pp. $103-122$. https://doi.org/10.1111/j.1460-2466.1999.tb02784.x

Secko, David M.; Tlalka, Stephany; Dunlop, Morgan; Kingdon, Ami; Amend, Elyse (2011). “The unfinished science story: Journalist-audience interactions from the Globe and Mail's online health and science sections". Journalism, v. 12, n. 7, pp. 814-831. https://doi.org/10.1177/1464884911412704

Shanahan, Marie-Claire (2010). "Changing the meaning of peer-to-peer? Exploring online comment spaces as sites of negotiated expertise". Journal of science communication, v. 9, n. 1, pp. 1-13.

https://doi.org/10.22323/2.09010201

Veltri, Giuseppe-Alessandro (2013). "Microblogging and nanotweets: Nanotechnology on Twitter". Public understanding of science, v. 22, n. 7, pp. 832-849.

http://ow.ly/PFzC30iRITR

https://doi.org/10.1177/0963662512463510

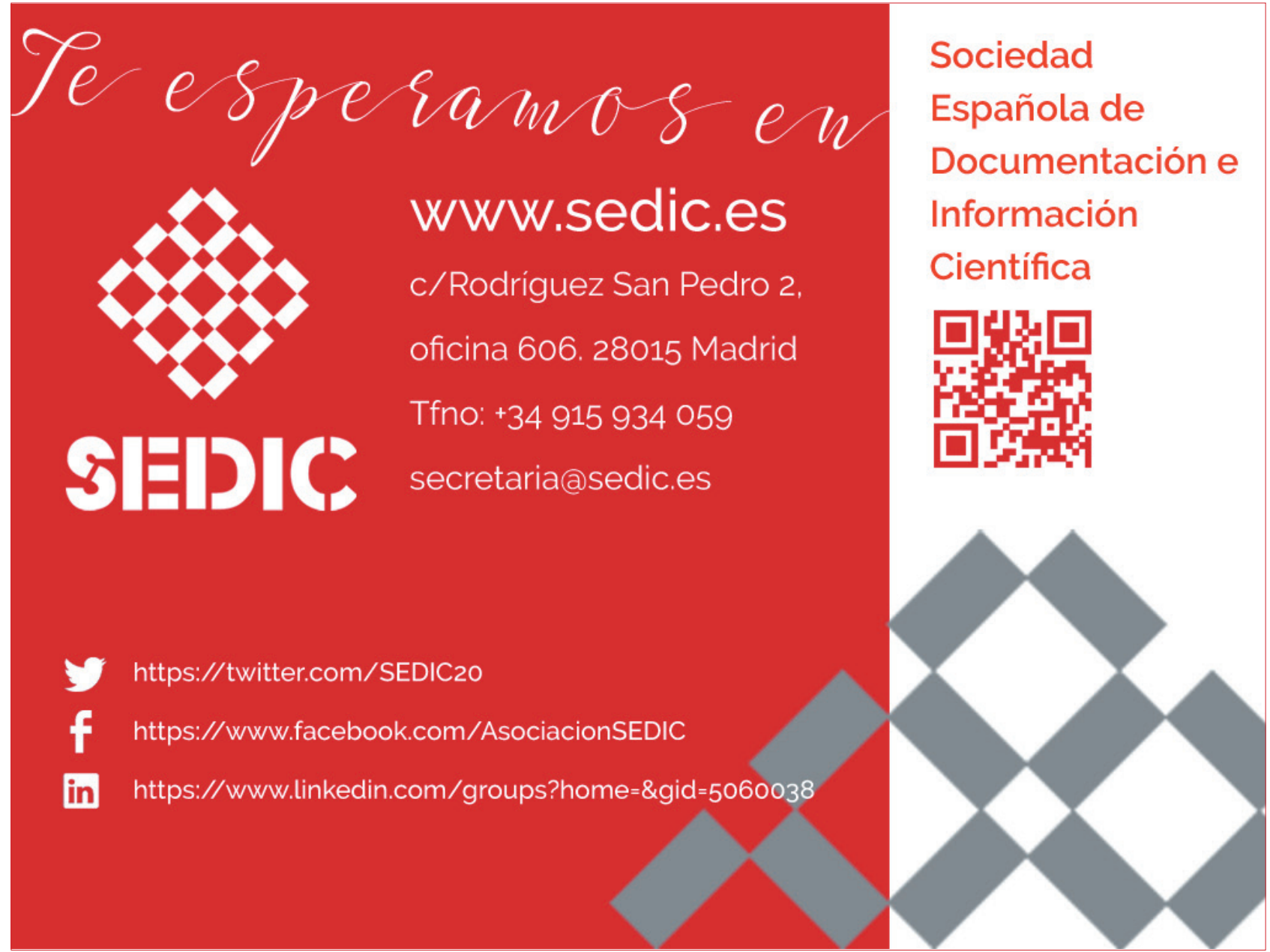

